\title{
Assurer la présence enseignante en formation à distance : des résultats de recherche pour guider la pratique en enseignement supérieur
}

Colette Deaudelin, Matthieu Petit et Louis Brouillette

\section{OpenEdition}

Journals

Édition électronique

URL : http://journals.openedition.org/trema/3411

DOI : 10.4000/trema.3411

ISSN : 2107-0997

Éditeur

Faculté d'Éducation de l'université de Montpellier

Édition imprimée

Date de publication : 1 mai 2016

Pagination : $79-100$

ISBN : 979-10-96627-00-4

ISSN : 1167-315X

\section{Référence électronique}

Colette Deaudelin, Matthieu Petit et Louis Brouillette, «Assurer la présence enseignante en formation à distance : des résultats de recherche pour guider la pratique en enseignement supérieur », Tréma [En ligne], 44 | 2016, mis en ligne le 01 juin 2016, consulté le 20 avril 2019. URL : http://

journals.openedition.org/trema/3411; DOI : 10.4000/trema.3411

Ce document a été généré automatiquement le 20 avril 2019

Trema 


\title{
Assurer la présence enseignante en formation à distance : des résultats de recherche pour guider la pratique en enseignement supérieur
}

\author{
Colette Deaudelin, Matthieu Petit et Louis Brouillette
}

1 En enseignement supérieur, les développements nombreux et importants sur le plan de la formation à distance (FAD) exigent que les personnes formatrices puissent s'appuyer sur les meilleures pratiques, guidées en cela par un repérage de connaissances issues de la recherche scientifique. Un tel repérage de connaissances en vue d'une mobilisation dans la formation à distance ou hybride ${ }^{1}$ est réalisé dans la recension qui suit.

2 Après avoir précisé le thème de notre recension au regard de défis de la $\mathrm{FAD}$, nous décrivons la méthode utilisée et en présentons les résultats. Enfin, une synthèse permet de dégager les principales pistes de travail à l'intention des personnes formatrices chargées de concevoir ou de dispenser un cours partiellement ou totalement à distance.

\section{Problématique}

3 Nombreuses sont les définitions de la FAD focalisant sur l'absence, c'est-à-dire sur la séparation physique de la personne formatrice et de l'étudiant. C'est précisément ce concept d'absence que retient Jacquenot en 1993 pour apprivoiser la distance, posant cette dernière comme fondement de la relation pédagogique en $\mathrm{FAD}$. La chercheuse définit d'ailleurs différentes distances, à savoir celles spatiale, temporelle, technologique, sociale, culturelle, économique et pédagogique pour conceptualiser toutes les absences induites par celles-ci. Jacquenot (1993) propose même un changement radical de vision conduisant non pas à supprimer l'absence mais plutôt à la considérer comme une qualité. Elle préconise en effet de mettre l'accent sur les points forts des dispositifs à distance de manière à les mettre au service de la formation en présentiel. Paquelin (2011), lui, 
circonscrit les proximités conduisant à la "bonne distance ", c'est-à-dire cet "espacetemps-social qui délimite et contient la zone d'activités participatives des sujets qui correspond à ce que ces acteurs sont en capacité de réaliser à la fois individuellement et collectivement pour répondre à un besoin» (p. 566). Cet auteur conclut que «l'engagement des apprenants dans le dispositif [de formation à distance] suppose l'ancrage de l'action dans un ensemble de proximités spatiales et non spatiales qui [favorise] l'élaboration d'un climat de confiance propice à l'initialisation d'un processus de formation » (p. 588).

4 Absence et proximité représentent donc deux construits intermédiaires conduisant au courant de recherches sur la présence en FAD. Peraya (2014) décrit d'ailleurs très bien cette évolution de la réflexion sur la présence et de concepts qui y sont associés. Plusieurs synthèses permettent notamment d'explorer les termes utilisés pour référer à la présence virtuelle (Kawachi, 2011) et de discuter de certains modèles comme celui de la communauté d'apprentissage en ligne ( $\left.\mathrm{CAL}^{2}\right)$ de Garrison et son équipe (voir Annand, 2011) ou encore d'en approfondir les fondements théoriques (Jezegou, 2010, 2012). D'autres recensions ont porté précisément sur la présence sociale ${ }^{3}$, sa conceptualisation (Oztok et Brett, 2011), l'évolution du concept (Cui, Lockee et Meng, 2013), ou encore sa mesure (Cui, 2013).

5 Toutefois, malgré la multiplication des travaux empiriques, très peu de revues systématiques des écrits permettent d'éclairer la pratique en FAD au regard du concept de présence. En effet, une recension dans des banques de données a permis de repérer 157 études scientifiques publiées depuis 2008 et portant sur différents types de présence, dont les présences sociale, enseignante, cognitive, apprenante et transactionnelle ${ }^{4}$. Ainsi à notre connaissance, seuls Denoyelles, Zydney et Chen (2014) ont réalisé une recension systématique d'études menées au cours des dix dernières années en focalisant toutefois sur celles répertoriées uniquement dans la base de données ERIC et sur le site web regroupant les travaux associés au modèle de CAL et en examinant seulement les études portant sur l'influence de stratégies spécifiques dans des cours complètement en ligne. Ce constat justifie la pertinence de mener une revue systématique des écrits en considérant, d'une part, un plus large ensemble de banques de données et, d'autre part, les recherches portant sur des cours en ligne mais aussi hybrides, étant donné la grande prévalence de cette modalité et les questions que l'amalgame présence et distance soulève.

Le corpus de 157 études que nous avons établi a conduit à orienter notre recension sur la présence la plus directement liée aux actions de la personne chargée de concevoir ou de dispenser une formation à distance, à savoir la présence enseignante, une présence qu'Arbaugh, Cleveland-Innes, Diaz, Garrison, Ice, Richardson et Swan (2008) jugent cruciale pour la satisfaction des étudiants et la réussite du processus d'enseignement et d'apprentissage.

7 Ainsi, notre recension vise à répondre à la question suivante : Quelles interventions la personne qui conçoit ou dispense un cours partiellement ou totalement à distance doitelle privilégier pour soutenir la présence en vue de favoriser l'apprentissage des étudiants? 


\section{Méthode}

8 La méthode est décrite en précisant le type de recen
ainsi que l'étendue et les limites de cette recension.

\section{II.1. Types de recension d'écrits}

9 Parmi les méthodes de recension décrites par Whitemore et Knafl (2005), on retrouve la recension intégrative, la recension systématique, la méta-analyse et la recension qualitative. Considérant la question de recherche posée, la recension systématique apparaît la plus pertinente. Utilisée dans le domaine de la santé pour synthétiser des résultats de recherche afin de répondre à une question précise soulevée par la pratique, elle apparaît donc adéquate pour guider celle en éducation.

\section{II.2. Corpus}

Dans le but de répondre à la question de recherche, la recension exhaustive d'articles scientifiques évoquée précédemment a été effectuée en tenant compte des critères suivants : articles évalués par les pairs, publiés entre 2008 et 2014, rédigés en anglais ou en français et traitant d'une forme de présence dans un ou des cours hybrides ou à distance en enseignement supérieur. Divers descripteurs, tant en français qu'en anglais, ont été choisis. Voici un exemple de descripteur pour chacune des catégories : elearning, teach* presence, higher education ${ }^{5}$.

11 La recherche d'articles a été menée dans les bases de données suivantes: Academic Search Complete, Cairn, CBCA Complete, Computers et Applied Sciences Complete, EdITLib, Education Source, ERIC, Érudit, Psychology et Behavioral Sciences Collection, PsycInfo, Repère, ScienceDirect et Scopus. Pour assurer une plus grande validité du corpus, une triangulation des sources a été effectuée en examinant les listes de références des articles déjà sélectionnés et de certains sites Internet ${ }^{6}$.

12 Afin de pouvoir exploiter des résultats à plus forte possibilité de généralisation, nous avons sélectionné les recherches utilisant des méthodes quantitatives ou mixtes ${ }^{7}$. Du corpus initial de 157 articles, 52 traitaient de façon quantitative ou mixte de la présence enseignante. Ce corpus a ensuite été réduit à 48 en raison de la faible pertinence de certaines études ou du manque de renseignements méthodologiques dans d'autres.

\section{II.3 Étendue et limites de la recension}

Les études quantitatives et mixtes sur la présence enseignante intègrent généralement d'autres types de présence ou encore des variables qui y sont associées, dont l'immédiateté, c'est pourquoi les relations avec ces autres éléments sont rapportées dans cet article. Par ailleurs, le choix de ne retenir que les recherches quantitatives ou mixtes sur la présence enseignante conduit à éliminer les recherches sur d'autres concepts développés pour traiter de la présence. Par exemple, Kawachi (2011) recense au moins six termes renvoyant à autant de présence. Outre les présences enseignante, cognitive et sociale, il relève également celles transactionnelle, institutionnelle et apprenante. 


\section{Résultats}

14 À des fins de concision, nous présentons d'abord globalement les cadres conceptuels et méthodologiques retenus; des informations relatives à chacune des études recensées sont compilées dans le tableau de l'annexe $1^{8}$. Ensuite, nous synthétisons les résultats de ces études.

\section{III.1 Cadres conceptuels et méthodologiques retenus}

15 Une majorité des études recensées exploitent le cadre conceptuel développé par l'équipe de Garrison à partir d'articles fondateurs (Anderson, Rourke, Garrison et Archer, 2001 ; Garrison, Anderson et Archer, 2000, 2001). Développé pour examiner les interactions dans le cadre de formations en ligne, le modèle de la communauté d'apprentissage en ligne (CAL) s'appuie sur la notion de practical inquiry de John Dewey de même que sur une approche constructiviste et collaborative de l'apprentissage. Ce cadre comprend trois éléments interdépendants et dynamiques, à savoir: les présences enseignante ( $\mathrm{PE})$, cognitive (PC) et sociale (PS). La PE peut être définie en tant que méthodes employées par les personnes formatrices pour créer des expériences d'apprentissage en ligne enrichissantes et stimulantes (Bangert, 2009). La PC renvoie, elle, à la capacité des étudiants à donner un sens à l'information qu'ils reçoivent et à le confirmer par des échanges au sein d'une communauté d'apprentissage (Garrison, Anderson et Archer, 2001) alors que la PS correspond à l'habileté qu'ont ces étudiants à communiquer leurs caractéristiques personnelles au sein d'une telle communauté et ainsi se présenter comme de réelles personnes (Garrison, Anderson et Archer, 2000).

La PE est mesurée à travers trois dimensions: le design et l'organisation du cours, la facilitation des échanges et l'enseignement. La PC comprend quatre phases d'un processus d'investigation, à savoir l'évocation d'éléments déclencheurs, l'exploration de solutions, l'intégration (qui correspond à la construction de solutions) et la résolution du problème posé. La PS s'opérationnalise à travers l'expression de l'affectivité, la communication ouverte et la cohésion du groupe. L'équipe de Shea a proposé une modélisation de la CAL intégrant la présence apprenante (Shea et Bidjerano, 2010, 2012 ; Shea, Hayes, Uzuner-Smith, Gozza-Cohen, Vickers et Bidjerano, 2014).

17 Les trois types de présence ont été souvent mesurés à travers un questionnaire validé comprenant 34 questions : 13 questions pour la PE, 9 pour la PS, et 12 pour la PC. Une analyse de contenu, utilisant l'approche de codage négocié élaborée par Garrison, Cleveland-Innes, Koole et Kappelman (2006, voir Akyol, Garrison et Ozden, 2009), est souvent utilisée pour l'examen des messages échangés dans les forums.

\section{III.2 Résultats des études recensées}

Deux sections rendent compte des principaux résultats statistiquement significatifs obtenus dans les études du corpus. La première regroupe celles établissant des relations entre les types de présence. La deuxième réunit les études où les types de présence agissent comme variables indépendantes (ou prédictives) ou comme variables dépendantes (ou critères). 


\section{III.2.1 Relations entre les présences enseignante, sociale et cognitive}

19 Garrison et des collaborateurs ont contribué à la validation du questionnaire à 34 énoncés mentionné précédemment ${ }^{10}$. Ils confirment la présence des trois facteurs et en démontrent la cohérence interne (Arbaugh et al., 2008 ; Swan, Richardson, Ice, Garrison, Cleveland-Innes et Arbaugh, 2008). Garrison, Cleveland-Innes et Fung (2010) ont confirmé que chaque énoncé du questionnaire n'est significatif que pour le type de présence auquel il est associé. L'étude de Diaz, Swan, Ice et Kupczynski (2010), qui tient compte non seulement de la perception qu'ont les étudiants de chacune des présences mais aussi de l'importance qu'ils y accordent, indique que ces derniers considèrent la PE plus importante que la PS et la PC. En plus de confirmer la validité conceptuelle du modèle théorique de la CAL, cette étude révèle deux dimensions de la PE : ce qui s'effectue avant le cours (le design et l'organisation) et ce qui se déroule durant le cours (la facilitation des échanges et l'enseignement).

20 Se penchant plus spécifiquement sur les relations entre les présences, une première étude de Shea et Bidjerano (2009a) montre que la PE et la PS sont corrélées à la PC alors qu'une seconde (2009b), confirmant la structure factorielle du modèle du CAL, indique que tant la PE que la PS ont un effet significatif sur la PC. De plus, en distinguant trois groupes de participants selon le niveau de PS perçue, Shea et Bidjerano (2009b) font ressortir le fait que la PS semble modérée par la PE dans l'influence exercée sur la PC. Ainsi, quel que soit le niveau de PS, une PE élevée aura un effet positif sur la PC.

21 Shea et des collègues (Shea, Hayes, Vickers, Gozza-Cohen, Uzuner, Mehta, Valchova et Rangan, 2010) identifient des corrélations entre les types de présence du modèle de la CAL en distinguant le formateur et l'étudiant : la PE du formateur est corrélée à la PS des étudiants, et la PS perçue par le formateur est corrélée à celle des étudiants. Cette deuxième corrélation est plus importante que la première.

L'étude de Garrison et al. (2010) ajoute à la compréhension du modèle en établissant que la PE perçue par les étudiants a un effet direct sur leur perception de la PC et qu'elle est associée de manière significative à la PS. L'étude de Joo, Lim et Kim (2011) démontre aussi que la PE a un effet significatif sur la PC et que la PE n'est pas seulement associée à la PS mais qu'elle a un effet sur cette dernière. Pour Hosler et Arend (2012), la PE explique 46,9 $\%$ de la variance de la PC et c'est sa composante relative à la facilitation des échanges qui a la plus grande contribution. Enfin, bien que Ke (2010) utilise un cadre théorique différent, elle obtient des résultats partiellement similaires puisqu'elle dégage de son analyse que la $\mathrm{PE}$, et plus spécifiquement le design du cours, prédit de façon significative la PS et la PC. Joo et al. (2011) démontrent de plus que la PS assure un effet médiateur sur la relation entre la PE et la PC, ce que confirme l'étude de Garrison et al. (2010).

En 2012, Shea et Bidjerano démontrent que la PS et la PE constituent des prédicteurs-clés des habiletés des apprenants les conduisant à une PC. L'apprentissage autorégulé, qui est synonyme de présence apprenante pour ces auteurs, a même été identifié en tant que variable modératrice des relations entre la PS, la PE et la PC.

Daspit et D'Souza (2012), examinant la contribution d'un wiki à une formation hybride, confirment les hypothèses formulées à savoir que, dans un tel environnement, la PE est positivement associée à la PC et la PS et que cette dernière l'est aussi positivement à la PC.

Enfin, Kozan et Richardson (2014) examinent à leur tour les relations entre les trois types de présence en contrôlant l'effet d'un type de présence pour examiner la relation entre 
les deux autres types, ce qui mène à des résultats différents de ceux présentés précédemment.

- La corrélation est importante et positive entre la PE et la PS, mais lorsqu'on retire l'effet de la PC, la relation entre la PE et la PS peut disparaitre. Ainsi, la PC est importante pour la relation entre la PE et la PS.

- La corrélation est importante et positive entre la PS et la PC. La corrélation entre PS et la PC semble indépendante à la PE. Ainsi, la PE aurait un effet limité sur la relation entre la PC et la PS.

- La corrélation est importante et positive entre la PE et la PC. La corrélation entre la PE et la PC semble indépendante de la PS. en ligne) n'a pas d'impact sur la relation entre la PE et la PC ${ }^{11}$. Gorsky, Caspi et Blau (2012) dans leur comparaison de deux institutions - l'une offrant de la formation à distance et l'autre en présentiel - dégagent les différences significatives suivantes :

- Chez les formateurs, la PE se manifeste principalement par des actions de design dans l'institution où la formation se déroule en présentiel, alors que pour l'autre, ce sont les interventions d'enseignement qui prévalent.

- Chez les étudiants, les différences se situent sur le plan de la PS où dans l'institution offrant la formation à distance, on retrouve plus de messages témoignant de l'expression d'affectivité et de communication ouverte. messages échangés dans les forums d'une étude d'Akyol et al. (2009) montre que le groupe 
en ligne a exprimé plus d'affectivité (PS) et davantage de messages servant à initier la discussion et à explorer des solutions (les deux premiers niveaux de la PC). Chez le groupe en formation hybride, un nombre significativement plus élevé de messages en lien avec la cohésion du groupe (PS) et avec l'intégration, soit le troisième niveau de PC, a été constaté. Les résultats significatifs obtenus montrent que les étudiants en formation hybride ont perçu une plus grande PE.

Les résultats de l'étude de Shea et Bidjerano (2010) vont dans le même sens en révélant que les étudiants suivant des cours hybrides ont perçu davantage de PE que ceux inscrits à des cours en ligne. De plus, la relation entre la perception qu'ont les étudiants de la PE et leur sentiment d'efficacité est plus forte chez les étudiants des cours hybride que chez ceux des cours en ligne. Les analyses de Shea et Bidjerano (2012) montrent aussi l'avantage des cours hybrides par rapport aux cours en ligne, car les étudiants y ont perçu davantage de PS, de PE et de PC. Aussi, l'étude de Shea et Bidjerano (2009b) révèle que dans les cours complètement en ligne, la qualité de la PE a un plus grand impact sur la PC que dans les cours hybrides.

Kucuk et Sahin (2013) ont sondé deux groupes d'étudiants : un groupe en présentiel et un autre dans une formule rendue hybride par l'utilisation de Facebook pour une partie du cours. À l'égard de la PE, aucune différence significative ne fut mesurée entre le cours en présentiel et sa version hybride.

Dans leur comparaison de formations exploitant soit la communication asynchrone, soit un amalgame d'asynchrone et de synchrone, Rockinson-Szapkiw, Baker, Neukrug, et Hanes (2010) démontrent que la seule différence statistiquement significative entre les deux groupes d'étudiants concerne la PS: ceux qui ont profité des deux modes de communication ont perçu davantage de PS que ceux qui ne bénéficiaient que du mode asynchrone. Dans sa recherche, Baker (2010) constate que les étudiants ayant un cours utilisant le mode asynchrone ont rapporté un niveau significativement plus bas de présence de l'enseignant ${ }^{12}$ et d'immédiateté $^{13}$ que ceux ayant un cours en mode synchrone.

\section{III.2.2.1.2 La dimension temporelle}

Akyolet Garrison (2008) ont exploré la dynamique développementale des trois types de présence au sein de CAL en distinguant trois périodes à la session d'études qui comptait neuf semaines (trois temps de trois semaines). Une analyse des dimensions de chacune des présences indique que les seules différences significatives concernent l'évolution de la PS et de la PE :

- pour la PS : une diminution de l'expression de l'affectivité et augmentation dans le temps des messages relatifs à la cohésion du groupe ;

- pour la PE : une croissance des messages liés à l'enseignement tout au cours de la session et une diminution de ceux visant à faciliter la discussion au cours des temps 1 et 2 .

Une autre équipe (Traver, Volchok, Bidjerano et Shea, 2014), utilisant une version du questionnaire de la CAL intégrant la présence apprenante (Shea et Bidjerano, 2010), a examiné le développement de la perception des présences. Entre le prétest et le posttest, les corrélations sont fortes et positives pour la PE, la PC et la PS, mais modérée pour la présence apprenante. Ainsi, la perception des étudiants quant aux PE, PC et PS tend à augmenter de manière significative au cours de la session. 
37 Akyol, Vaughan et Garrison (2011) ont examiné l'impact de la durée de la formation en ligne (6 ou 13 semaines) sur le développement de la CAL et des trois types de présence. Leurs résultats indiquent que :

- le groupe participant à une session de 13 semaines a envoyé davantage de messages 1) exprimant l'affectivité (PS), 2) associés à l'enseignement (PE) et 3) témoignant des deux plus hauts niveaux de la PC (intégration et résolution);

- le groupe participant à une session de 6 semaines a envoyé plus de messages témoignant de la cohésion du groupe (PS) et de la phase d'exploration de l'investigation (PC). Il s'est dit plus satisfait, considère avoir appris davantage et a perçu une plus grande PE.

\section{III.2.2.1.3 L'abandon ou la rétention} étudiants de son cours lorsque ce nombre avoisine les 200. Chacune des propositions de recherche des étudiants est alors évaluée par deux autres étudiants du cours. Les évaluations sont à leur tour évaluées par celui qui les reçoit. Cette initiative pédagogique a permis d'augmenter le taux de réussite du cours de $17 \%$ en un an seulement. De plus, la PE a été le type de présence qui a reçu le score moyen le plus élevé par les étudiants du cours (soit 4,31 sur 5 pour la PE ; 4 pour la PC et 3,52 pour la PS); la dimension de la PE 
recevant la plus haute cote a été le design et l'organisation du cours avec une moyenne de 4,67 .

Bangert (2008), quant à lui, a vérifié l'effet de deux types d'intervention sur les messages échangés dans un forum : une intervention soutenant la PS, et une autre axée autant sur la PS et que la PE. Le groupe soutenu uniquement sur le plan de la PS a envoyé un nombre significativement plus élevé de messages agissant comme déclencheurs (1er niveau de la $\mathrm{PC})$. Le groupe ayant bénéficié d'interventions axées sur la PS et la PE a envoyé une proportion significativement plus grande de messages des deux plus hauts niveaux de la $\mathrm{PC}$, à savoir les niveaux d'intégration et de résolution.

Gorsky, Caspi, Antonovsky, Blau et Mansur (2010), supposant des modes d'interventions différents selon les champs disciplinaires (sciences exactes et sciences humaines), ont vérifié l'impact de cette variable sur les échanges dans des forums. Ils ont constaté un ratio constant de messages témoignant des types de présence, à savoir quelque $60 \%$ pour la PS, $20 \%$ pour la PC et $20 \%$ pour la PE, et ce peu importe la discipline ${ }^{15}$. Toutefois, l'analyse d'Arbaugh, Bangert et Cleveland-Innes (2010) révèle que la PE, la PS et la PC sont perçues de façon significativement plus élevée par les étudiants en sciences appliquées qu'en sciences pures.

Dans l'étude d'Arbaugh (2010), le temps moyen de connexion des formateurs par session de travail sur le site du cours prédit négativement la perception qu'ont les étudiants de leurs apprentissages. L'auteur arrive à la conclusion contre-intuitive que plus la personne formatrice consacre du temps au design et à l'organisation du cours pendant (plutôt qu'avant) les activités pédagogiques avec ses étudiants, moins elle est efficace.

Rockinson-Szapkiw (2012) a comparé deux groupes de doctorants, l'un recevant des rétroactions écrites et audio ${ }^{16}$ et l'autre recevant exclusivement des commentaires écrits. Bien que les scores moyens indiquent qu'il n'y a pas de différence significative entre les deux groupes au sujet de la PS, elle montre que les étudiants qui ont reçu les rétroactions écrites et audio sur leurs devoirs ont rapporté des niveaux plus élevés de PE et de PC que les étudiants qui ont reçu seulement les commentaires écrits.

Miller et Redman (2010) ont mesuré la PE perçue par les étudiants dans un cours d'astronomie en ligne lorsque des vidéos ont été utilisées pour diverses démonstrations traditionnellement faites en classe. Une majorité d'étudiants a trouvé les vidéos efficaces pour la création de PE dans un environnement en ligne. Une forte corrélation a été mise en évidence tant entre la PE et la PS qu'entre la PE et la PC. L'attitude des étudiants envers le cours s'est révélée aussi fortement corrélée à leur perception de la PE.

L'étude de Rubin, Fernandes et Avgerinou (2013), portant sur les perceptions des étudiants face à des systèmes de gestion des apprentissages (comme Blackboard, DesireToLearn et Moodle) et leur influence sur la CAL, montre que la perception qu'ont des étudiants en ce qui a trait aux possibilités qu'offre la technologie - en particulier sur le plan de la communication - prédit tant la PE, la PS que la PC.

50 Par ailleurs, la facilité d'utilisation du système de gestion des apprentissages n'a un effet significatif que sur la PE, et cet effet est négatif.

51 L'éclairage qu'apportent ces études va amener la personne formatrice à :

- favoriser un cours de format hybride plutôt que totalement en ligne ;

- choisir un amalgame de communications asynchrone et synchrone ou de diverses technologies soutenant la communication ; 
- privilégier une formation s'échelonnant sur une durée normale (13 semaines) plutôt qu'intensive (6 semaines) afin d'atteindre de plus hauts niveau de PC ;

- se rappeler que la communication claire des éléments importants du cours, tout en guidant la compréhension de ceux-ci, favorise la rétention des étudiants ;

- respecter un délai de réponse annoncé ;

- mettre à profit la contribution des étudiants et les assister dans leurs différents rôles, comme celui de procurer de la rétroaction;

- considérer que la perception de présence dans les forums ne semble pas influencée par les particularités des champs disciplinaires concernés.

\section{III.2.2.2 L'effet des types de présence sur des dimensions cognitives et affectives de l'apprentissage ${ }^{17}$} indiquent une corrélation entre la PE et les résultats scolaires des étudiants. Les scores moyens obtenus dans l'étude de Rockinson-Szapkiw (2012) montrent pour leur part que les doctorants ayant reçu des commentaires écrits et audio pour leurs devoirs ont perçu avoir davantage appris et ils ont obtenu des notes plus élevées que les étudiants qui ont seulement reçu des commentaires écrits. perçus. Toutefois, seules la PE et la PS sont positivement corrélées à la satisfaction des étudiants. Akyolet Garrison (2008) ont, quant à eux, constaté des relations significatives entre les trois types de présence et la satisfaction des étudiants et, en ce qui a trait à l'apprentissage perçu, des corrélations ont été notées uniquement avec la PE et la PC. ker (2010), lui, s'est penché sur deux variables prédictives (la présence de l'enseignan et son immédiateté ${ }^{18}$ ) et une variable cognitive, qui correspond à la mesure de ce que l'étudiant croit avoir appris dans le cours par rapport à ce qu'il pourrait avoir appris avec le formateur idéal. L'analyse indique que la présence de l'enseignant et son immédiateté expliquent $46 \%$ de la variance de la variable cognitive, $56 \%$ de la variance de l'attitude de l'étudiant à l'égard du cours et $38 \%$ de sa motivation. prédicteurs de la perception qu'ont des étudiants de leurs apprentissages, la PE étant toutefois la variable prédictive la plus importante. La même étude indique que la PE se révèle une variable prédictive significative de la satisfaction des étudiants à l'égard du support médiatique sur lequel se donnent les cours en ligne. des étudiants, la PC ayant la plus grande contribution (50,5\%).

Constatant le niveau d'appréciation très différent de deux groupes d'étudiants sous la responsabilité de deux formateurs, Gorsky et Blau (2009) ont noté, en ne considérant que les messages des formatrices, les différences significatives suivantes :

- un plus grand nombre de messages provenait de la formatrice la plus appréciée ;

- le temps de réponse de cette dernière était huit fois moins long que celui de la formatrice la moins appréciée, celle-ci ayant même négligé de répondre à près du quart des messages qui lui ont été envoyés ;

- la formatrice la plus appréciée intervenait plus fréquemment pour faciliter le discours et pour fournir un enseignement (deux dimensions témoignant de la PE), soutenait la PC par 
un plus grand nombre de messages stimulant l'intégration des solutions et la résolution du problème, et envoyait plus de messages pour soutenir la PS. l'engagement des étudiants suivant des cours en ligne. Le modèle utilisé, différent de la CAL, est celui de Roblyer et Wiencke (2004, voir Lear et al., 2009) qui comporte cinq éléments: 1- planification de l'interaction sociale, 2- planification des activités d'apprentissage, 3- technologies utilisées pour l'interaction, 4- signes de l'engagement du formateur, et 5- signes de l'engagement de l'apprenant. Les quatre premiers éléments de la rubrique, qui relèvent $\mathrm{du}$ formateur, s'avèrent significatifs pour l'engagement de l'apprenant.

Bien que peu d'études établissent clairement la relation entre la PE et les résultats scolaires, les études rapportées ici confirment pour la personne formatrice l'importance de ses actions de design, de facilitation et d'enseignement puisque sa présence prédit la satisfaction des étudiants et permet d'expliquer la variation de leur motivation et de leur attitude ainsi que la perception qu'ils ont de leurs apprentissages.

\section{Comment soutenir la présence enseignante pour améliorer la performance des étudiants?}

Cette dernière section comprend une synthèse des résultats présentés précédemment et intègre certains résultats qualitatifs des recherches exploitant des méthodes mixtes. De plus, des liens sont établis avec les résultats de la recension systématique menée par Denoyelles et al. (2014). Cette synthèse vise à guider la personne qui conçoit ou dispense un cours partiellement ou totalement à distance dans le choix des interventions qu'elle doit privilégier pour soutenir les divers types de présence et ainsi améliorer l'apprentissage des étudiants.

Bien que les résultats rapportés ne soient pas consensuels, des lignes directrices peuvent être dégagées. Alors que le modèle de la CAL bénéficie d'un intérêt renouvelé, le rôle capital de la PE ressort grandement des études. Lear et al. (2009) considèrent même le formateur comme l'élément le plus significatif pour le développement d'un lien d'appartenance entre les étudiants membres d'une communauté d'apprentissage par la structure qu'il donne au cours et les interactions qu'il suscite. Les répondants de l'étude de Diaz et al. (2010), considèrent la PE plus importante que la PS et la PC. Ainsi, la PE est associée à la PC (Shea et Bidjerano, 2009a). Elle prédit même la PC (Garrison et al., 2010; Joo et al., 2011; Shea et Bidjerano, 2009b), en explique la variance (Hosler et Arend, 2012) ou encore modère la relation PS-PC (Shea et Bidjerano, 2009b). Bien que peu d'études du corpus étudié portent spécifiquement sur les environnements médiatisés exploités, la PE conserve un rôle important. Par exemple, elle est associée à la PC dans un environnement wiki (Daspit et D'Souza, 2012) et peut être perçue tout autant dans un cours utilisant Facebook qu'elle ne l'est en présentiel (Kucuk et Sahin, 2013).

Outre ces relations, la PE entretient des liens d'association ou de causalité avec d'autres variables, comme l'apprentissage perçu par les étudiants (dont Arbaugh, 2008; Akyolet Garrison, 2008), leurs résultats scolaires (Shea, Hayes et Vickers, 2010), leur satisfaction (notamment Akyolet Garrison, 2008; Arbaugh, 2008; Hosler et Arend, 2012), leur motivation ou plus largement leur attitude à l'égard d'un cours (Baker, 2010), ce qui confirme son rôle capital. 
63 Le design s'impose comme une dimension importante de la PE, car selon Ke (2010), elle prédit la PC et la PS. Cette relation devra être validée, car l'étape de design étant généralement faite préalablement à la formation, les études recensées recèlent peu de données la concernant. Arbaugh (2010) conclut d'ailleurs à l'importance que le design du cours soit effectivement fait avant le déroulement de la formation.

Les participants consultés souhaitent que :

- le cours soit bien organisé (Hosler et Arend, 2012);

- les travaux soient pertinents et leur but, clairement présenté (Hosler et Arend, 2012) ;

- des occasions de se familiariser avec les tâches soient planifiées et leur soient offertes (Traphagan, Chiang, Chang, Wattanawaha, Lee, Mayrath, Woo, Yoon, Jee et Resta, 2010).

- Des résultats des études recensées montrent que les actions suivantes du formateur facilitent le discours :

- des questions pertinentes, ouvertes qui agissent comme déclencheurs (Hosler et Arend, 2012) et qui exigent des réponses en profondeur (Bangert, 2008) ;

- des réponses qui mettent l'accent sur les perspectives différentes chez les étudiants et qui modélisent les processus cognitifs complexes (Bangert, 2008);

- une orchestration des échanges par le formateur (Ke, 2010).

65 Au sujet des actions de facilitation, en conclusion Denoyelles et al. (2014) soulignent que celles-ci doivent mettre de l'avant un protocole d'échanges et demeurer étroitement liées au but général de la discussion et aux objectifs spécifiques d'apprentissage.

S'exprimant sur l'enseignement, des répondants mentionnent qu'ils souhaitent des rétroactions rapides et spécifiques (Hosler et Arend, 2012) ; signifiantes et procurant un soutien individuel (Ke, 2010); et clarifiant leurs conceptions erronées (Bangert, 2008). Denoyelles et al. (2014) concluent toutefois que les rétroactions de la personne formatrice doivent demeurer modestes pour encourager celles des étudiants qui doivent en conserver le leadership.

Bien que les résultats la concernant soient moins consensuels que ceux relatifs à la $\mathrm{PE}$, le rôle de la PS ne doit pas être négligé. De l'avis de Shea et Bidjerano (2012), la PS serait même à la base des interactions en ligne chez les étudiants. La PS se révèle, elle aussi, tantôt une variable associée à la PC (Shea et Bidjerano, 2009a, 2009b), tantôt une variable prédictrice de l'autorégulation de l'apprentissage (Shea et al., 2014), tantôt variable médiatrice de la relation PE-PC (Garrison et al., 2010 ; Joo et al., 2011). Deris, Zakaria et Mansor (2012) considèrent pour leur part que la présence personnelle du formateur joue un rôle pivot dans les trois composantes de la PE. Au sujet de la façon dont la PS se manifeste, Ladyshewsky (2013) rapporte que les étudiants apprécient davantage un formateur qui reconnait leur contribution, personnalise ses messages en utilisant leur prénom, utilise des expressions laissant transparaître son sens de la communauté et exprime de la gratitude à leur égard. Ces résultats, qui montrent l'importance pour les formateurs de modeler des interventions assurant une PS, convergent avec ceux de Denoyelles et al. (2014), auteurs qui au terme de leur recension arguent qu'il faut accroitre la PS.

Le rôle de l'étudiant, partiellement décrit par les éléments du sondage concernant la PC, est défini différemment par les auteurs recensés. Par exemple, Nagel et Kotze (2010) utilisent l'évaluation par les pairs pour pallier la difficulté d'assurer une PE suffisante dans des classes pléthoriques alors que d'autres mettent de l'avant une stratégie où il y a dévolution $d u$ rôle $d u$ formateur sur les étudiants, stratégie prévoyant une première 
étape de modelage de la facilitation des échanges. L'autorégulation des apprentissages, ayant mené Shea et Bidjerano $(2010,2012)$ à circonscrire la présence apprenante, représente une avenue guidant la définition du rôle de l'étudiant dans une CAL. Les résultats de l'étude de Coll, Engel et Bustos (2009) confirment d'ailleurs que la PE ne s'exerce pas uniquement par le formateur dans un cours en ligne donné de manière asynchrone. Torras et Mayordomo (2011) observent de leur côté une corégulation entre formateur et étudiants. Denoyelles et al. (2014) concluent également que les étudiants peuvent assumer certains rôles habituellement dévolus au formateur, ce qui permet à ce dernier d'axer davantage ses interventions sur le savoir d'expertise qu'il détient.

Même si l'abandon en FAD demeure un problème difficile à cerner (Ice et al., 2011 ; Traver et al., 2014), les environnements les plus riches semblent proposer des pistes de solutions, entre autres en amenant les étudiants à percevoir davantage de présence. Pensons à la richesse technologique d'un environnement 3D dans l'étude de Burgess, Slate, RojasLebouef et Laprairie (2010), au format hybride qui tire profit d'un volet en présentiel (Akyol et al., 2009; Shea et Bidjerano, 2010), à l'amalgame de modes de communication asynchrone et synchrone (Rockinson-Szapkiw et al., 2010) ou de médias (l'écrit et l'audio chez Rockinson-Szapkiw, 2012, et chez Dringus, Snyder et Terrell, 2010, et la vidéo chez Miller et Redman, 2010). Deris et al. (2012) montrent, eux, l'intérêt de multiplier les outils de communication entre le formateur et les étudiants. Toutefois, Lambert et Fisher (2013) recommandent de ne pas faire appel à trop de nouvelles technologies, car le défi pour les étudiants sera alors d'apprivoiser celles-ci. Traitant de la rétroaction, Denoyelles et al. (2014) soulignent au terme de leur recension que le recours à plusieurs médias accroit la perception de différentes présences.

Le temps joue également un rôle important pour que la CAL puisse révéler son plein potentiel. Il contribue à l'évolution positive de la perception des types de présence (Traver et al., 2014), où les formations s'inscrivant dans une durée normale mènent les étudiants vers les plus niveaux de PC (Akyol et al., 2011). Du côté de la rétroaction, ce sont les courts délais de réponse du formateur qui sont appréciés par les étudiants (Skramstad et al., 2012). Hodges et Cowan (2012), proposent que les délais de réponse soient annoncés aux étudiants. Enfin, sous le concept d'immédiateté, transparaît une dimension de l'interaction renvoyant à un éventail de conduites verbales et non verbales de l'enseignant visant à réduire la distance psychologique et physique en situation de formation dont l'importance commence à être étudiée. Notons que cette dimension temporelle ne ressort pas de la recension de Denoyelles et al. (2014).

\section{Conclusion}

71 La recension réalisée sur la PE contribue à préciser et réaffirmer le rôle de la personne formatrice en contexte de formation à distance ou hybride. Shea et Bidjerano (2009b) considèrent même que la qualité pédagogique telle qu'elle s'exprime par la PE et la PS constitue un facteur important dans la prédiction d'un apprentissage signifiant quel que soit l'âge de l'apprenant alors qu'Arbaugh (2010) précise que la PE doit être établie en premier afin de former une CAL. Cette recension apporte également un éclairage sur le rôle des étudiants dans ces nouveaux contextes de formation, en suggérant certaines avenues prometteuses, dont l'autorégulation de l'apprentissage. Comme le notent Shea et Bidjerano (2010), celle-ci peut compenser une faible PE. Toutefois, l'abandon des étudiants en $\mathrm{FAD}$ demeure un problème à examiner. 
72 Les limites conceptuelles et méthodologiques de la présente recension ou inhérentes aux études analysées constituent autant d'avenues de recherche. Rappelons d'abord le choix de recenser les études traitant au moins de la $\mathrm{PE}$, ce qui a conduit à négliger certains articles ne portant que sur d'autres types de présence. Sur le plan méthodologique, des clarifications sont nécessaires aux dires de Swan et al. (2008), la PS des formateurs étant prise en compte dans la PE alors que Ke (2010) montre plutôt que celle-ci est considérée de façon distincte de la PE. Ke (2010) de même que Shea, Hayes et Vickers (2010) questionnent la seule utilisation des échanges dans le forum pour mesurer la PC. Enfin, les futures recherches devront mieux tenir compte de l'apprentissage des étudiants par des mesures non autorapportées.

73 Sur le plan conceptuel, des travaux semblent incontournables afin de faire évoluer le modèle de la CAL. Ceux de Shea et al. (2014) ajoutent la présence apprenante. Jezegou (2010) cible la notion de transaction issue du pragmatisme et l'approche européenne du socioconstructivisme. Elle met à l'avant-plan la collaboration contradictoire insistant ainsi sur l'expression de divergences et la confrontation de points de vue. En 2012, Jezegou propose un modèle tridimensionnel de la présence représentant l'influence des trois dimensions (présences socio-affective, pédagogique et sociocognitive) sur la présence globale, modèle où la $\mathrm{PC}$ devient la présence sociocognitive pour bien marquer son ancrage dans le socioconstructivisme. Enfin, Kawachi (2011) ouvre sur d'autres présences qu'il importe d'examiner comme la présence transactionnelle qui vise à distinguer le soutien social à des fins d'apprentissage ou non ou encore la présence institutionnelle qui permet de considérer des dimensions macro liées à l'institution.

Annexe 1 : tableau des études recensées

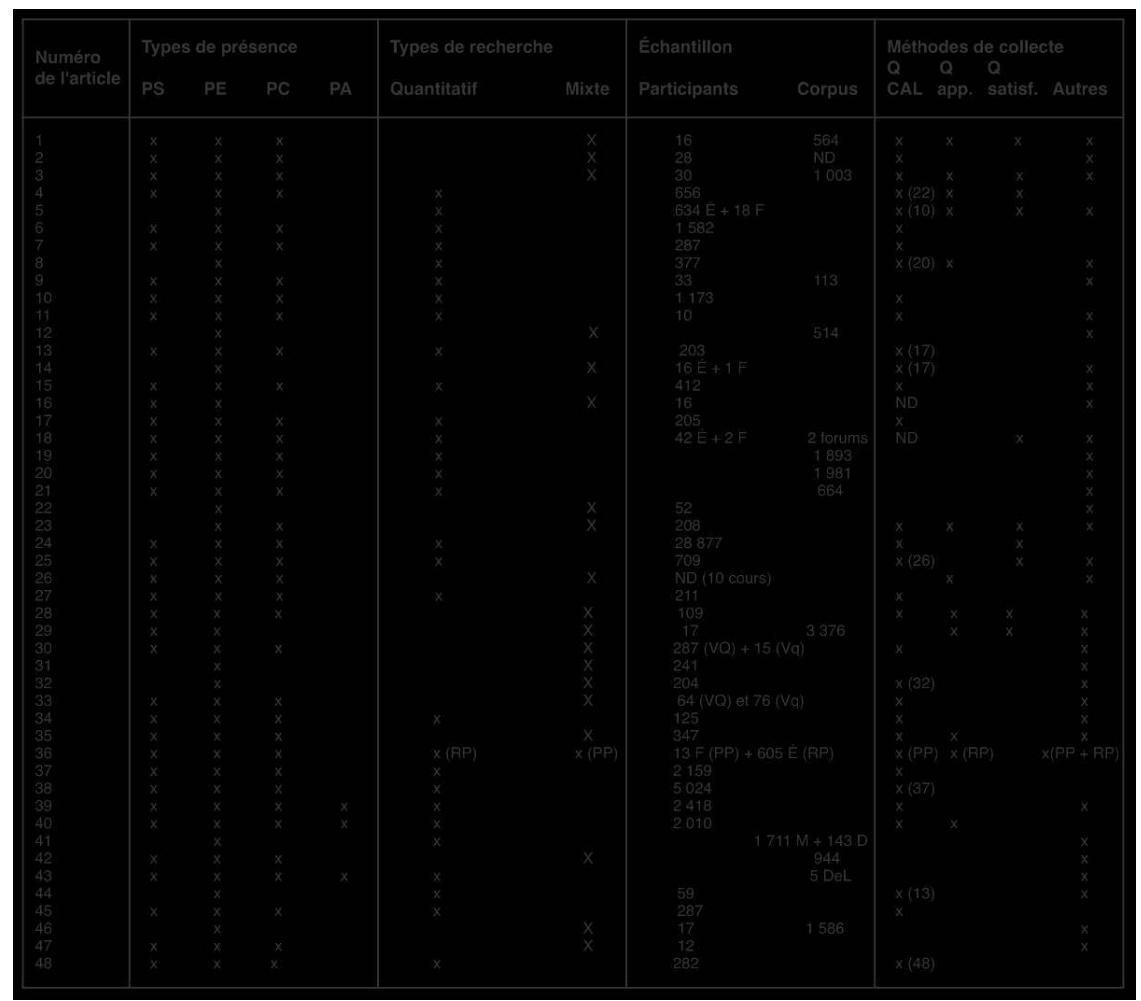


74 Note: implicitement, les participants sont des étudiants et le corpus est formé de messages. Dans la colonne «Q CAL », le nombre de questions posées est précisé lorsque le questionnaire de la CAL utilisé ne comporte pas 34 éléments. Légende :

App. Apprentissages perçus

D : Document

DeL : Discussion en ligne

$\mathrm{E}$ : étudiant

$\mathrm{M}$ : Message

ND : Non disponible

PA : Présence apprenante

F : Formateur

PP : Projet pilote

$\mathrm{RP}$ : Recherche principale

Q: Questionnaire

Satis. : Satisfaction

$\mathrm{Vq}$ : Volet qualitatif

$\mathrm{VQ}$ : volet quantitatif

\section{BIBLIOGRAPHIE}

Akyol, Z. and Garrison, D. R. (2008), « The development of a community of inquiry over time in an online course: Understanding the progression et integration of social, cognitive andteaching presence », in Journal of Asynchronous Learning Networks, vol. 12, nº 3: 3-22. [1]

Akyol, Z. and Garrison, D. R. and Ozden, M. Y. (2009), « Online and blended communities of inquiry: Exploring the developmental and perceptional differences ", in International Review of Research in Open and Distance Learning, vol. 10, n6. [2]

Akyol, Z. ; Vaughan, N. and Garrison, D. R. (2011), « The impact of course duration on the development of a community of inquiry ", in Interactive Learning Environments, vol. 19, nº 3 : 231-246. [3]

Anderson, T., Rourke, L., Garrison, D. R. and Archer, W. (2001), « Assessing teaching presence in a computer conferencing context », in Journal of Asynchronous Learning Network, vol. 5, nº 2 .

Annand, D. (2011), « Social presence within the community of inquiry framework », in The International Review of Research in Open and Distance Learning, vol. 12, nº $5: 40-56$.

Arbaugh, J. B. (2008), « Does academic discipline moderate CoI-course outcomes relationships in online MBA courses ? ", in International Review of Research in Open et Distance Learning, vol. 9, no 2. [4]

Arbaugh, J. B. (2010), « Sage, guide, both, or even more? An examination of instructor activity in online MBA courses ", in Computers and Education, vol. 55, nº 3: 1234-1244. [5] 
Arbaugh, J. B., Bangert, A.W. and Cleveland-Innes, M. (2010), « Subject matter effects et the community of inquiry (CoI) framework: An exploratory study », in The Internet and Higher Education, vol. 13, ํㅡ1-2: 37-4. [6]

Arbaugh, J. B., Cleveland-Innes, M., Diaz, S. R., Garrison, D. R., Ice, P., Richardson, J. C. and Swan, K. P. (2008), « Developing a community of inquiry instrument: Testing a measure of the community of inquiry framework using a multi-institutional sample », in The Internet and Higher Education, vol. 11, no 3-4: 133-136. [7]

Baker, C. (2010), « The impact of instructor immediacy et presence for online student affective learning, cognition, and motivation », in Journal of Educators Online, vol. 7, nํ $1: 1-30$. [8]

Bangert, A. W. (2008), « The influence of social presence et teaching presence on the quality of online critical inquiry ", in Journal of Computing in Higher Education, vol. 20, nº $1: 34-61$. [10]

Bangert, A.W.(2009), «Building a validity argument for the community of inquiry survey instrument », in The Internet and Higher Education, vol. 12, no 2: 104-111. [9]

Burgess, M. L., Slate, J. R., Rojas-Lebouef, A. and Laprairie, K (2010), « Teaching et learning in second life: Using the community of inquiry (CoI) model to support online instruction with graduate students in instructional technology ", in The Internet and Higher Education, vol. 13, no $1-2: 84-88$. [11]

Coll, C., Engel, A. and Bustos, A. (2009), « Distributed teaching presence et participants' activity profiles: A theoretical approach to the structural analysis of asynchronous learning networks ", in European Journal of Education, vol. 44, no 4 : 521-538. [12]

Cui, G. (2013), « Evaluating online social presence: An overview of social presence assessment », in Educational Technology Development and Exchange, vol. 6, nº $1: 13-30$.

Cui, G., Lockee, B. and Meng, C. (2013), « Building modern online social presence: A review of social presence theory and its instructional design implications for future trend ", in Education and Information Technologies, vol. 18, nº 14: 661-685.

Daspit, J. J. and D'Souza, D. E. (2012), « Using the community of inquiry framework to introduce wiki environments in blended-learning pedagogies: Evidence from a business capstone course », in Academy of Management Learning and Education, vol. 11, $\mathrm{n}^{\circ}$ 4: 666-683. [13]

Denoyelles, A., Zydney, J. M. and Chen, B. (2014), « Strategies for Creating a Community of Inquiry through Online Asynchronous Discussions », in Journal of Online Learning and Teaching, vol. 10, $\mathrm{n}^{\mathrm{o}}$ 1: 153-165.

Deris, F. D., Zakaria, M. H. and Mansor, W. F. A. W.(2012), « Teaching presence in online course for part-time undergraduates ", in Procedia - Social and Behavioral Sciences, vol. 66, nº 7 : 255-266. [14]

Diaz, S. R., Swan, K., Ice, P and Kupczynski, L. (2010), « Student ratings of the importance of survey items, multiplicative factor analysis, and the validity of the community of inquiry survey ", in The Internet and Higher Education, vol. 13 nº 1-2 : 22-30. [15]

Dringus, L. P. and Snyder, M. M. and Terrell, S. R. (2010), « Facilitating discourse et enhancing teaching presence: Using mini audio presentations in online forums ", in The Internet and Higher Education, vol. 13, no 1-2: 75-77. [16]

Garrison, D. R., Anderson, T. and Archer, W. (2000), « Critical inquiry in a text-based environment: Computer conferencing in higher education ", in The Internet and Higher Education, vol. 2, nº 2-3: 87-105. 
Garrison, D. R., Anderson, T. and Archer, W. (2001), « Critical thinking, cognitive presence, and computer conferencing in distance education », in American Journal of Distance Education, vol. 15, no 1: 7-23.

Garrison, D. R., Cleveland-Innes, M. and Fung, T. S. (2010), « Exploring causal relationships among teaching, cognitive and social presence: Student perceptions of the community of inquiry framework », in The Internet and Higher Education, vol. 13, no 1-2 : 31-64. [17]

Gorsky, P. and Blau, I. (2009), « Online teaching effectiveness : A tale of two instructors », in International Review of Research in Open and Distance Learning, vol. 10, $\mathrm{n}^{\circ}$ 3. [18]

Gorsky, P., Caspi, A. , Antonovsky, A., Blau, I. and Mansur, A. (2010), « The relationship between academic discipline and dialogic behavior in open university course forums », in International Review of Research in Open and Distance Learning, vol. 11, nº 2 : 49-72. [19]

Gorsky, P., Caspi, A. , Blau, I. (2012), « A comparison of non-mandatory online dialogic behavior in two higher education blended environments ", in Journal of Asynchronous Learning Networks, vol. 16, no 4 : 55-69. [20]

Gorsky, P., Caspi, A. , Blau, I., Vine, Y. and Billet, A. (2012), « Toward a CoI population parameter: Impact of unit (sentence vs. message) on the results of quantitative content analysis ", in International Review of Research in Open and Distance Learning, vol. 13, no $1: 17-37$. [21]

Hodges, C. B. and Cowan, S. F. (2012), « Preservice teachers' views of instructor presence in online courses », in Journal of Digital Learning in Teacher Education, vol. 28, no 4 : 139-145. [22]

Hosler, K. A. and Arend, B. D. (2012), « The importance of course design, feedback, and facilitation: Student perceptions of the relationship between teaching presence and cognitive presence », in Educational Media International vol. 49, no 3: 217-29. [23]

Ice, P., Gibson, A. M., Boston, W. and Becher, D. (2011), « An exploration of differences between community of inquiry indicators in low and high disenrollment online courses ", in Journal of Asynchronous Learning Networks, vol. 15, no 2 : 44-69. [24]

Jacquenot, G. (1993), « Apprivoiser la distance et supprimer l'absence ? Ou les défis de la formation à distance », in Revue française de pédagogie, nº 102 : 55-67.

Jézégou, A. (2010), «Créer de la présence à distance en e-learning : Cadre théorique, définition, et dimensions clés », in Distances et savoirs, vol. 8, nº $2: 257-74$.

Jézégou, A. (2012), «La présence en e-learning : Modèle théorique et perspectives de recherche », in La Revue internationale de l'apprentissage en ligne et de l'enseignement à distance, vol. 26, nº 1 .

Joo, Y. J., Lim, K. Y. and Kim, E. K. (2011), « Online university students' satisfaction and persistence: Examining perceived level of presence, usefulness and ease of use as predictors in a structural model », in Computers and Education, vol. 57, nº $2:$ 1654-1664. [25]

Kawachi, P. (2011), « Unwrapping presence », in Distances et savoirs, vol. 9, nº 4 : 591-609.

Ke, F. (2010), « Examining online teaching, cognitive, and social presence for adult students », in Computers and Education, vol. 55, nº 2: 808-20. [26]

Kozan, K. and Richardson, J. C. (2014), « Interrelationships between and among social, teaching, and cognitive presence », in The Internet and Higher Education, vol. $21: 68-73$. [27]

Kucuk, S. and Sahin, I. (2013), « From the perspective of community of inquiry framework: An examination of facebook uses by pre-service teachers as a learning environment ", in Turkish Online Journal of Educational Technology, vol. 12, nº 2: 142-156. [28] 
Ladyshewsky, R. K. (2013), « Instructor presence in online courses and student satisfaction », in International Journal for the Scholarship of Teaching et Learning, vol. 7, $\mathrm{n}^{\circ}$ 1. [29]

Lambert, J.L. and Fisher, J. L. (2013), « Community of inquiry framework: Establishing community in an online course ", in Journal of Interactive Online Learning, vol. 12, nº 1: 1-16. [30]

Lear, J. L., Isernhagen, J. C., Lacost, B. A. and King, J. W. (2009) « Instructor presence for webbased classes », in Delta Pi Epsilon Journal, vol. 51, no 2 : 86-98. [31]

Miller, S. and Redman, S. (2010), «Improving instructor presence in an online introductory astronomy course through video demonstrations », in Astronomy Education Review, vol. 9, ㄲo 1. [32]

Nagel, L. and Kotzé, T. G. (2010), « Supersizing e-learning: What a CoI survey reveals about teaching presence in a large online class ", in The Internet and Higher Education, vol. 13, nำ 1-2: 45-51. [33]

Oztok, M. and Brett, C. (2011), « Social presence and online learning: A review of research », in Revue de l'Éducation à Distance, vol. 25, no 3.

Paquelin, D. (2011), « La distance : Question de proximités », in Distances et Savoirs, vol. 9, no 4 : 565-590.

Peraya, D. (2014), « Distances, absence, proximités et présences : Des concepts en déplacement », in Distances et médiations des savoirs, vol. 8.

Rockinson-Szapkiw, A. J., (2012), «Should online doctoral instructors adopt audio feedback as an instructional strategy? Preliminary evidence ", in International Journal of Doctoral Studies, vol. 7: 245-258. [34]

Rockinson-Szapkiw, A. J., Baker, J. D., Neukrug, E. and Hanes, J. (2010), « The efficacy of computer mediated communication technologies to augment and support effective online helping profession education », in Journal of Technology in Human Services, vol. 28, no $3:$ 161-77. [35]

Rubin, B., Fernandes, R. and Avgerinou M. D. (2013), « The effects of technology on the community of inquiry and satisfaction with online courses ", in The Internet and Higher Education, vol. 17: 48-57. [36]

Shea. P. and Bidjerano, T. , (2009b) « Cognitive presence and online learner engagement : A cluster analysis of the community of inquiry framework », in Journal of Computing in Higher Education, vol. 21, no 3 : 199-217. [37]

Shea, P. and Bidjerano, T., (2009a) « Community of inquiry as a theoretical framework to foster "epistemic engagement" and "cognitive presence" in online education ", in Computers and Education, vol. 52, nº 3: 543-553. [38]

Shea, P. and Bidjerano, T., (2010), « Learning presence: Towards a theory of self-efficacy, selfregulation, and the development of a communities of inquiry in online and blended learning environments », in Computers and Education, vol. 55, no 4: 1721-1731. [40]

Shea, P. and Bidjerano, T., (2012), « Learning presence as a moderator in the community of inquiry model », in Computers and Education, vol. 59, nำ 2: 316-326. [39]

Shea, P., Hayes, S., Uzuner-Smith, S., Gozza-Cohen, M., Vickers, J. and Bidjerano, T. (2014), « Reconceptualizing the community of inquiry framework: Exploratory and confirmatory analysis ", in The Internet and Higher Education, vol. 23: 9-17.[41]

Shea, P., Hayes, S. and Vickers, J. (2010), « Online instructional effort measured through the lens of teaching presence in the community of inquiry framework: A re-examination of measures and 
approach ", in International Review of Research in Open and Distance Learning, vol. 11, nº 3 :127-54.

[42]

Shea, P., Hayes, S., Vickers, J., Gozza-Cohen, M., Uzuner, S., Mehta, R., Valchova, A. and Rangan, P. (2010), «A re-examination of the community of inquiry framework : Social network and content analysis », in The Internet and Higher Education, vol. 13, nº 1-2 : 10-21. [43]

Skramstad, E, Schlosser, C. and Orellana, A. (2012), « Teaching presence and communication timeliness in asynchronous online courses ", in Quarterly Review of Distance Education, vol. 13, ํㅜ 3: 183-188. [44]

Swan, K. P., Shea, P., Richardson, J. C., Ice, P., Garrison, D. R., Cleveland-Innes, M., Arbaugh, J. B. (2008), « Validating a measurement tool of presence in online communities of inquiry », in $E$ mentor, vol. 24, $\mathrm{n}^{\circ} 2$. [45]

Torras, M. E., Mayordomo, R. (2011), « Teaching presence and regulation in an electronic portfolio ", in Computers in Human Behavior, vol. 27, n 6: 2284-2291. [46]

Traphagan, T. W., Chiang, Y-H. V., Chang, H. M., Wattanawaha, B, Lee, H., Mayrath, M. C., Woo, J, Yoon, H. J., Jee, M. J. and Resta, P. E. (2010), « Cognitive, social and teaching presence in a virtual world and a text chat », in Computers and Education, vol. 55, $\mathrm{n}^{\circ}$ 3: 923-936. [47]

Traver, A. E., Volchok, E., Bidjerano, T. and Shea, P. (2014), « Correlating community college students' perceptions of community of inquiry presences with their completion of blended courses ", in The Internet and Higher Education, vol. 20 :1-9. [48]

Whitemore, R. and Knafl, K. (2005), « The integrative review: Updated methodology », in Journal of Advanced Nursing, vol. 52, $\mathrm{n}^{\circ}$ 5: 546-53.

\section{NOTES}

1. Généralement définie comme une formation alliant présentiel et distance.

2. Traduction que nous faisons de la Community of inquiry.

3. Voir la section III.1 pour la définition de ce concept.

4. Des précisions sur cette recension se trouvent à la section II.2.

5. Les traductions appropriées ont été utilisées pour chacune des catégories de descripteurs, dont les exemples suivants : elearning, teach* presence, higher education.

6. Dont le suivant: https://coi.athabascau.ca/

7. Largement définies comme les recherches regroupant les études alliant des méthodes qualitatives et quantitatives.

8. Le tableau renvoie aux auteurs par des chiffres qui permettent d'identifier l'article recensé dans la bibliographie.

9. Voir études à la section III.2.1.

10. Dans la suite de l'article, nous ne préciserons le questionnaire mesurant les présences que lorsqu'il sera différent de celui à 34 énoncés.

11. Aucun impact non plus en considérant des variables comme l'âge, le sexe ou le nombre de cours en ligne déjà suivis.

12. Il est à noter que, bien que Baker s'appuie sur Shea, Li et Pickett (2006), qui utilisent le modèle de la CAL, l'auteur indique étudier la présence de l'enseignant plutôt que la présence enseignante.

13. Baker (2010) définit ainsi le concept d'immédiateté : «L'immédiateté de la communication [... ] renvoie aux conduites verbales et non verbales qui réduisent la distance psychologique et physique entre des individus » (traduction libre, p. 4). 
14. Traduction libre de 2 des 34 énoncés du questionnaire.

15. Gorsky, Caspi, Blau, Vine et Billet (2012) démontrent que l'unité d'analyse, à savoir la phrase plutôt que le message, n'a pas d'impact sur cette distribution des messages entre les trois types de présence.

16. Enregistrés avec Audacity ou Acrobat Adobe 9.

17. Nous considérons ici uniquement les études où la mesure de l'apprentissage est distincte de celle de la PC.

18. Voir la note 11 .

\section{RÉSUMÉS}

En enseignement supérieur, les personnes engagées dans la formation à distance désirent s'appuyer sur les meilleures pratiques. L'article fait état d'une recension des recherches scientifiques portant principalement sur la présence enseignante. L'analyse de 52 études recourant à des méthodes quantitatives ou mixtes a conduit à l'identification de lignes directrices concernant les présences enseignante et sociale, le rôle de l'étudiant et divers aspects des environnements de formation.

In higher education, those involved in distance education want to rely on best practices. The article reports a literature review mostly on the teaching presence. The analysis of 52 studies using quantitative or mixed methods led to the identification of guidelines for teaching and social presences, the role of the student and various aspects of training environments.

\section{INDEX}

Mots-clés : communauté d'apprentissage en ligne, formation à distance, présence cognitive, présence enseignante, présence sociale, recension systématique d'écrits.

Keywords : cognitive presence, community of inquiry, distance education, social presence, systematic research review., teaching presence

\section{AUTEURS}

\section{COLETTE DEAUDELIN}

Ph. D., Professeure titulaire, Faculté d'éducation, Université de Sherbrooke

\section{MATTHIEU PETIT}

Ph. D., Professeur, Faculté d'éducation, Université de Sherbrooke

\section{LOUIS BROUILLETTE}

Ph. D., Professionnel de recherche, Faculté d'éducation, Université de Sherbrooke 